\title{
A global perspective on the costs of hypertension: a systematic review
}

\author{
Ewelina Wierzejska ${ }^{1}$, Bogusz Giernaś ${ }^{1}$, Agnieszka Lipiak ${ }^{1}$, Monika Karasiewicz $^{1}$, Mateusz Cofta ${ }^{1}$, \\ Rafał Staszewski
}

\begin{abstract}
'Department of Preventive Medicine, Laboratory of International Health, Poznan University of Medical Sciences, Poznan, Poland ${ }^{2}$ Department of Hypertensiology, Angiology and Internal Medicine, Laboratory of Pharmacoeconomics in Hypertension, Poznan University of Medical Sciences, Poznan, Poland
\end{abstract}

Submitted: 10 September 2019

Accepted: 9 December 2019

Arch Med Sci 2020; 16 (5): 1078-1091

DOI: https://doi.org/10.5114/aoms.2020.92689

Copyright $\odot 2020$ Termedia \& Banach

\begin{abstract}
Introduction: Hypertension, particularly untreated, leads to serious complications and contributes to high costs incurred by the whole society. The aim of the review was to carry out a social and economic comparison of various categories of hypertension costs from different countries.

Material and methods: The study was a systematic review. PubMed, Cochrane Library and Google Scholar databases were searched. Hypertension costs were analyzed in 8 cost categories. An attempt was made to determine whether selected economic and social factors (such as HDI or GDP) influenced hypertension costs.

Results: The review included data from 15 countries: Brazil, Cambodia, Canada, China, Greece, Indonesia, Italy, Jamaica, Kyrgyzstan, Mexico, Poland, Spain, USA, Vietnam and Zimbabwe. The papers included in the review were heterogeneous with respect to cost categories, which made comparisons difficult. The average total costs of hypertension for all the studied countries, calculated per person, amounted to 630.14 Int\$, direct costs - 1,497.36 Int\$, and indirect costs $-282.34 \mathrm{Int}$. The ranking of countries by costs and by selected economic and social indices points at the possible relationship between these indices and hypertension costs.

Conclusions: The costs of hypertension calculated per country reached the region of several dozen billion Int\$. Other sources usually showed lower costs than those presented in this review. This indicates a growth in costs from year to year and the future increasing burden on society. Globally uniform cost terminology and cost calculation standards need to be developed. That would facilitate making more informed decisions regarding fund allocation in hypertension management schemes.
\end{abstract}

Key words: hypertension costs, total costs, direct costs, indirect costs, hospital costs, drug costs, socioeconomic indices.

\section{Introduction}

Cardiovascular diseases (CVDs) are the leading cause of morbidity and death globally. Hypertension ( $\mathrm{HT})$, together with dyslipidemia, is a major risk factor for CVDs [1], regardless of sex or ethnicity [2]. The etiology of high blood pressure is varied. With prevalence from $20 \%$ to even

\author{
Corresponding author: \\ Ewelina Wierzejska MPH, PhD \\ Department \\ of Preventive Medicine \\ Laboratory \\ of International Health \\ Poznan University \\ of Medical Sciences \\ 6 Święcickiego St \\ 60-781 Poznan, Poland \\ E-mail: mailto:ewierz@ump. \\ edu.pl
}


$50 \%$ in adults, HT is the most common condition in developed countries [3]. It is usually defined as blood pressure exceeding 140/90 $\mathrm{mm} \mathrm{Hg}$ [4]. This definition was adopted for the purposes of this article as valid within the time scope covered in the review. However, according to the most recent guideline for hypertension of the American College of Cardiology (ACC), hypertension is defined as blood pressure exceeding 130/80 $\mathrm{mm} \mathrm{Hg}$ [2].

As a risk factor for CVDs, HT is indirectly the leading cause of death and the third cause of disability globally [5]. Its long-term effects include damage to such organs as blood vessels, heart, and kidneys [6]. Frequent HT complications are left ventricular hypertrophy [7], heart failure [8], and stroke [9].

The aim of this study was to collect and compare data on the costs of hypertension management, morbidity, and mortality across different countries and across different cost categories. An attempt was made to adopt a global perspective, including cost data from as many countries in the world as possible. The aim of this approach was to identify the categories where the costs are the highest. Such information would allow for better planning of future healthcare spending, for example through changes in fund allocation or reorganization of hypertension care focused on primary prevention. Consequently, this could contribute to more cost-effective hypertension management. Additionally, the authors tried to go beyond the cost analysis and adopt a socio-economic perspective with the use of indices commonly used in international rankings.

The cost data come from original research papers analyzing the phenomenon for a particular country or a region of a country (such as Quebec in Canada or particular states in Mexico). HT costs were divided into the following categories: total costs - the sum of direct and indirect costs, direct costs connected with HT therapy (drugs, medical equipment, diagnostics, hospitalization, consultations, nursing, medical transport, etc.), indirect costs, i.e. economic losses caused by decreased productivity of employees (presenteeism), their absence from work caused by sickness (absenteeism) and premature deaths, hospital costs, i.e. those related specifically to hospitalization, drug costs, i.e. the costs related to the purchase and management of medications, pharmaceutical care costs, i.e. costs related to pharmacist's participation in the treatment, out-of-pocket expenses, i.e. the expenses paid directly by the patient, and finally costs of stroke as a separate category of direct costs. Such cost classification and definitions are commonly used in pharmacoeconomic analyses, for instance in the study by Mitchell-Fearon et al. [10]. Since stroke is a frequent complication of HT, the costs of stroke were also included in the study. As was noted by Turin et al., the lifetime risk of stroke for men at the age of 45 was $17.21 \%$ for men without HT and $32.79 \%$ for hypertensive men [11].

\section{Material and methods}

According to the categories presented above, HT costs were calculated and presented in tables, either on a per patient basis or as a total. Additional analyses were carried out as regards cost structure. Finally, countries were ranked by costs and by selected socioeconomic indices in order to check whether an increase of costs coincided with an increase of particular indices.

\section{Literature search}

The search was carried out in PubMed, Cochrane and Google Scholar. In each case, the following search terms were used: hypertension costs, hypertension economic burden, hypertension economic impact, hypertension economic effects and hypertension social effects. The terms were searched for in both titles and abstracts. The following filters were used in PubMed: free full texts available, a publication date from 1 January 2014 to 31 December 2018, and English as the language of publication.

The filters used in Cochrane Library were: a trial as the type of study, and a publication date from 2014 to 2018.

In the Google Scholar database, the only filter used was a publication date from the year 2014 onwards. The default sorting by relevance was retained.

Out of the publications found, all of the results from PubMed and Cochrane Library databases were taken into account. By contrast, only the first 100 pages of search results from Google Scholar (sorted by relevance) were taken into account, as further pages presented results irrelevant to the search terms. In addition, reference lists of included publications were also searched manually to be able to include also paid publications from journals subscribed by our institution.

Selection was carried out by two authors independently in accordance with the PRISMA Statement [12]. First, duplicate records and articles not related to HT costs were excluded. Next, the authors excluded articles not available as the full text, those which were not original research, and those where the presented costs were not comparable. Studies based on forecasting (e.g. Markov modeling) were included on the assumption that the models used permit reliable cost estimates. Subsequently, the quality of the articles was assessed using the Downs and Black 


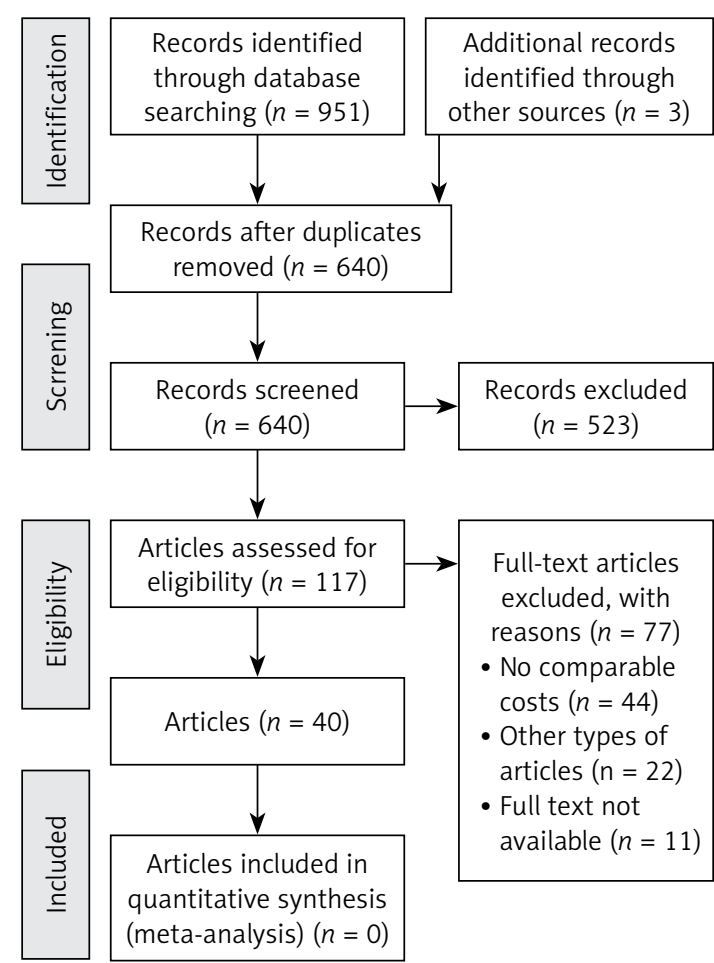

Figure 1. PRISMA flow diagram showing the record selection process

checklist [13]. As a result, 40 publications were included in the review. Since the data in the selected studies turned out not to be homogeneous enough, no meta-analysis was conducted (Figure 1).

\section{Synthesis of literature}

The following information on the included articles was collated: the name of the main author, country or region described, study population, type of research, reference year for costs, and cost currency. All the extracted cost information was allocated to a specific cost category and, in the few cases where the cost was not given in international dollars (Int\$), conversion was carried out. The exchange rates for this currency were taken from the databases of the World Bank [14] for the year given as a reference year for costs in a given study. If the reference year was not specified, it was assumed that the cost data refer to the year when a given study was accepted for publication. Amounts in local currencies were given using ISO 4217 currency codes. As the exchange rate for international dollars in relation to a local currency is always calculated on the basis of the purchasing power parity of the United States dollar (USD), the Int\$/USD exchange rate always equals 1 . In the case of a few Polish studies where costs were given in euro (EUR) despite the official Polish currency being the Polish zloty (PLN), the Int\$/EUR exchange rate in Germany in a given year was used.

\section{Results}

When assessed for data quality (very low, low, moderate, high), 22 of the 40 publications presented data of low quality, 17 presented data of moderate quality, and 1 presented data of high quality. There were no publications with very low quality of data. The quality of a majority of publications was on the borderline between low and moderate.

Table I presents all the publications included in the review [10, 15-53]. Table II shows HT costs - divided into comparable categories - extracted from the publications included in the review.

The lowest cost values were observed 9 times in Indonesia, 6 times in Jamaica, 3 times in Brazil, twice in Mexico, once in Kyrgyzstan, once in the USA, and once in Vietnam. The highest cost values were observed 15 times in the USA, twice in Jamaica, twice in Canada, once in Brazil, once in Greece, once in Spain, and once in Poland (Table III).

On average, direct costs turned out to constitute a slightly bigger share $(51.5 \%)$ of the total costs than indirect costs (48.5\%). Generally, it can be assumed that the proportion of one type of costs to the other does not exceed $60 \%$ vs. $40 \%$. Such analysis of cost structure was carried out for only four countries, since only in these cases were all the necessary data (i.e. total costs, direct costs and indirect costs) available (Table IV).

On average, the biggest share in the cost structure is that of other costs (53.33\%) and hospital costs $(26.33 \%)$, while the smallest share is that of drug costs (20.33\%). A possible reason for the considerable differences in the cost structure between the analyzed countries is, apart from the specific character of each country, the different methodology of gathering component data. This analysis was carried out for only three countries for which all the necessary data (i.e. direct costs, hospital costs and drug costs) were available (Table V).

Additional socio-economic data are presented in Tables VI and VII.

\section{Discussion}

No reviews were found with a scope so wide as the scope of this review. The only study covering more than one country was that by Mullins 2004 [54], which compared hospital costs and outpatient treatment costs for HT complications, including stroke. Therefore, the data from this review were compared mainly to studies covering individual countries.

In one study carried out in the USA (Benjamin 2019) [55], total HT costs were estimated at $55,900,000,000$ USD, direct costs at 51,300,000,000 
Table I. Details of the included studies

\begin{tabular}{|c|c|c|c|c|c|}
\hline Record & $\begin{array}{l}\text { Country } \\
\text { (region) }\end{array}$ & Population & Type of study & $\begin{array}{l}\text { Reference } \\
\text { year for costs } \\
\text { (currency) }\end{array}$ & $\begin{array}{l}\text { Purchasing } \\
\text { power } \\
\text { parity }\end{array}$ \\
\hline $\begin{array}{l}\text { Alvarez-Sabin } \\
2016[15]\end{array}$ & Spain & $\begin{array}{l}176 \text { aged over } \\
18 \text { years, mean } \\
\text { age } 72.1 \text { years } \\
\text { (54.8\% males) }\end{array}$ & $\begin{array}{l}\text { Epidemiological, } \\
\text { observational, prospective, } \\
\text { multicenter study }\end{array}$ & 2012 (EUR) & $\begin{array}{l}\operatorname{lnt} \$ 1= \\
0.695 \text { EUR }\end{array}$ \\
\hline $\begin{array}{l}\text { Arredondo } \\
2015[16]\end{array}$ & Mexico & $\begin{array}{l}654701 \\
\text { reported cases } \\
\text { of adults with } \\
\text { hypertension }\end{array}$ & Cost estimation & 2015 (USD) & - \\
\hline $\begin{array}{l}\text { Arredondo } \\
2016 \text { [17] }\end{array}$ & $\begin{array}{l}\text { Mexico (Hidalgo, } \\
\text { Jalisco, Morelos, } \\
\text { Sinaloa, Yucatán) }\end{array}$ & Unspecified & Time series study & 2015 (USD) & - \\
\hline $\begin{array}{l}\text { Bernard } 2014 \\
{[18]}\end{array}$ & USA & $\begin{array}{c}9383 \text { aged } \\
18-64 \text { years } \\
(49.9 \% \text { males })\end{array}$ & $\begin{array}{l}\text { Study based on data from } \\
\text { the Medical Expenditure } \\
\text { Panel Survey - Household } \\
\text { Component (MEPS - HC) }\end{array}$ & 2009 (USD) & - \\
\hline $\begin{array}{l}\text { Bigdeli } 2016 \\
\text { [19] }\end{array}$ & Cambodia & $\begin{array}{l}1250 \text { aged over } \\
18 \text { years, mean } \\
\text { age } 56 \text { years } \\
(33.0 \% \text { males })\end{array}$ & $\begin{array}{l}\text { Cross-sectional household } \\
\text { survey }\end{array}$ & 2014 (USD) & - \\
\hline $\begin{array}{l}\text { Boan } 2014 \\
{[20]}\end{array}$ & USA & $\begin{array}{c}84179 \text { aged } \\
\text { over } 25 \text { years } \\
(35.5 \% \text { blacks, } \\
37.0 \% \text { aged } \\
\text { over } 65 \text { years) }\end{array}$ & $\begin{array}{l}\text { Study based on data from } \\
\text { the State Inpatient Hospital } \\
\text { Discharge Database }\end{array}$ & 2014 (USD) & - \\
\hline $\begin{array}{l}\text { Boubouch- } \\
\text { airopoulou } \\
2014[21]\end{array}$ & Greece & $\begin{array}{l}116 \text { aged over } \\
30 \text { years }\end{array}$ & Costs estimation & 2014 (EUR) & $\begin{array}{l}\operatorname{lntS} 1= \\
0.611 \text { EUR }\end{array}$ \\
\hline $\begin{array}{l}\text { Bueno } 2017 \\
{[22]}\end{array}$ & Brazil & $\begin{array}{c}806 \text { aged } \\
\text { over } 60 \text { years } \\
(39.1 \% \text { males })\end{array}$ & Cross-sectional study & 2015 (USD) & - \\
\hline $\begin{array}{l}\text { Cazarim } 2018 \\
{[23]}\end{array}$ & Brazil & $\begin{array}{c}104 \text { aged } \\
38-83 \text { years }\end{array}$ & Cost-effectiveness study & 2015 (USD) & - \\
\hline $\begin{array}{l}\text { Finkelstein } \\
2014[24]\end{array}$ & Indonesia & $\begin{array}{l}10795 \text { mean } \\
\text { age } 53.3 \text { years } \\
(47.8 \% \text { males })\end{array}$ & $\begin{array}{c}\text { Study based on data from } \\
\text { Indonesian Family Life Survey } \\
\text { (IFLS) }\end{array}$ & 2008 (Int\$) & - \\
\hline $\begin{array}{l}\text { Gilmer } 2014 \\
{[25]}\end{array}$ & USA & $\begin{array}{c}71617 \text { aged } \\
3-17 \text { years } \\
(49.0 \% \text { males })\end{array}$ & $\begin{array}{l}\text { Prospective dynamic cohort } \\
\text { study }\end{array}$ & 2010 (USD) & - \\
\hline $\begin{array}{l}\text { Huang } 2016 \\
{[26]}\end{array}$ & China & $\begin{array}{c}21925 \text { mean } \\
\text { age } 61.05 \text { years } \\
\text { (48.7\% males) }\end{array}$ & $\begin{array}{c}\text { Study based on data from } \\
\text { National Health Services } \\
\text { Utilization Survey }\end{array}$ & 2016 (CNY) & $\begin{array}{l}\operatorname{lnt} \$ 1= \\
3.473 \mathrm{CNY}\end{array}$ \\
\hline $\begin{array}{l}\text { Kawalec } 2015 \\
{[27]}\end{array}$ & Poland & $\begin{array}{l}74745 \text { mean } \\
\text { age } 59.7 \text { years } \\
(41.5 \% \text { males })\end{array}$ & Cost estimation & 2014 (PLN) & $\begin{array}{l}\operatorname{lnt} \$ 1= \\
1.767 \text { PLN }\end{array}$ \\
\hline $\begin{array}{l}\text { Kirkland } 2018 \\
{[28]}\end{array}$ & USA & $\begin{array}{l}83018 \text { aged } \\
18-85 \text { years } \\
(45.6 \% \text { males })\end{array}$ & $\begin{array}{c}\text { Study based on data from } \\
\text { Medical Expenditure Panel } \\
\text { Survey }\end{array}$ & 2016 (USD) & - \\
\hline $\begin{array}{l}\text { Lekoubou } \\
2018[29]\end{array}$ & USA & $\begin{array}{c}7421 \text { aged } \\
18-85 \text { years } \\
(45.4 \% \text { males })\end{array}$ & $\begin{array}{c}\text { Study based on data } \\
\text { from Medical Expenditure } \\
\text { Panel Survey Household } \\
\text { Component }\end{array}$ & 2016 (USD) & - \\
\hline Liu 2016 [30] & China & $\begin{array}{l}414 \text { mean age } \\
64.3 \text { years } \\
(34.1 \% \text { males })\end{array}$ & $\begin{array}{l}\text { Study based on data from } \\
\text { Cross-sectional surveys }\end{array}$ & 2015 (CNY) & $\begin{array}{l}\operatorname{lnt} \$ 1= \\
3.478 \mathrm{CNY}\end{array}$ \\
\hline
\end{tabular}


Table I. Cont.

\begin{tabular}{|c|c|c|c|c|c|}
\hline Record & $\begin{array}{l}\text { Country } \\
\text { (region) }\end{array}$ & Population & Type of study & $\begin{array}{l}\text { Reference } \\
\text { year for costs } \\
\text { (currency) }\end{array}$ & $\begin{array}{c}\text { Purchasing } \\
\text { power } \\
\text { parity }\end{array}$ \\
\hline $\begin{array}{l}\text { Marra } 2017 \\
{[31]}\end{array}$ & Canada & $\begin{array}{c}\text { Mean age } \\
63.5 \text { years } \\
(48.8 \% \text { males })\end{array}$ & Cost-effectiveness study & 2015 (CAD) & $\begin{array}{c}\text { Int\$ } 1= \\
1.247 \text { CAD }\end{array}$ \\
\hline $\begin{array}{l}\text { Milan } 2017 \\
{[32]}\end{array}$ & Canada (Quebec) & $\begin{array}{c}881 \text { aged } \\
\text { over } 65 \text { years } \\
(36.6 \% \text { males })\end{array}$ & $\begin{array}{c}\text { Study based on data from } \\
\text { "Étude sur la santé des } \\
\text { aînés" }\end{array}$ & 2017 (CAD) & $\begin{array}{c}\operatorname{lnt} \$ 1= \\
1.251 \mathrm{CAD}\end{array}$ \\
\hline $\begin{array}{l}\text { Mitchell- } \\
\text { Fearon } 2017 \\
\text { [10] }\end{array}$ & Jamaica & $\begin{array}{c}2934 \text { aged } \\
\text { over } 60 \text { years } \\
(47.9 \% \text { males })\end{array}$ & Cost estimation & 2012 (USD) & - \\
\hline $\begin{array}{l}\text { Moise } 2016 \\
{[33]}\end{array}$ & USA & $35-74$ years & Cost estimation & 2016 (USD) & - \\
\hline $\begin{array}{l}\text { Murphy } 2016 \\
{[34]}\end{array}$ & Kyrgyzstan & $\begin{array}{c}9109 \\
\text { (47.7\% males) }\end{array}$ & $\begin{array}{l}\text { Study based on data from } \\
\text { Kyrgyzstan Integrated } \\
\text { Household Survey (KIHS) }\end{array}$ & 2016 (USD) & - \\
\hline $\begin{array}{l}\text { Mutowo } 2016 \\
{[35]}\end{array}$ & Zimbabwe & $\begin{array}{c}344 \text { mean age } \\
58.6 \text { years } \\
(36.3 \% \text { males })\end{array}$ & $\begin{array}{l}\text { Retrospective study based on } \\
\text { secondary data from medical } \\
\text { records }\end{array}$ & 2016 (USD) & - \\
\hline $\begin{array}{l}\text { Nguyen } 2014 \\
{[36]}\end{array}$ & Vietnam & $\begin{array}{c}230 \text { mean age } \\
64.3 \text { years } \\
(46.5 \% \text { males })\end{array}$ & Retrospective study & 2011 (USD) & - \\
\hline $\begin{array}{l}\text { Paczkowska } \\
2014 \text { [37] }\end{array}$ & Poland & $\begin{array}{c}480 \text { aged } \\
16-18 \text { years } \\
(69.4 \% \text { males })\end{array}$ & Retrospective study & 2013 (EUR) & $\begin{array}{l}\operatorname{lnt} \$ 1= \\
0.775 \text { EUR }\end{array}$ \\
\hline $\begin{array}{l}\text { Park } 2017 \\
{[38]}\end{array}$ & USA & $\begin{array}{c}26049 \text { aged } \\
\text { over } 18 \text { years } \\
(49.0 \% \text { males })\end{array}$ & $\begin{array}{c}\text { Study based on data from } \\
\text { Medical Expenditure Panel } \\
\text { Survey }\end{array}$ & 2014 (USD) & - \\
\hline $\begin{array}{l}\text { Ricci } 2017 \\
{[39]}\end{array}$ & Italy & Unspecified & Cost estimation & 2011 (EUR) & $\begin{array}{l}\operatorname{lnt} \$ 1= \\
0.759 \text { EUR }\end{array}$ \\
\hline $\begin{array}{l}\text { Ritchey } 2016 \\
{[40]}\end{array}$ & USA & Over 18 years & $\begin{array}{l}\text { Study based on data from } \\
\text { IMS Health's National } \\
\text { Prescription Audit }\end{array}$ & 2015 (USD) & - \\
\hline $\begin{array}{l}\text { Shireman } \\
2016[41]\end{array}$ & USA & $\begin{array}{l}494 \text { mean } \\
\text { age } 53.5 \text { years } \\
(35 \% \text { males, } \\
100 \% \text { blacks })\end{array}$ & Cost-effectiveness study & 2015 (USD) & - \\
\hline $\begin{array}{l}\text { Specogna } \\
2017[42]\end{array}$ & Canada & $\begin{array}{l}987 \text { mean } \\
\text { age } 72 \text { years } \\
(55 \% \text { males })\end{array}$ & Cohort study & 2017 (USD) & - \\
\hline $\begin{array}{l}\text { Stafylas } 2015 \\
{[43]}\end{array}$ & Greece & $\begin{array}{c}\text { Mean age } \\
53.2 \text { years } \\
(55.3 \% \text { males })\end{array}$ & Cost-utility study & 2013 (EUR) & $\begin{array}{l}\operatorname{lnt} \$ 1= \\
0.631 \text { EUR }\end{array}$ \\
\hline $\begin{array}{l}\text { Tajeu } 2017 \\
{[44]}\end{array}$ & USA & $\begin{array}{c}30239 \text { over } \\
45 \text { years mean } \\
\text { age } 66.8 \text { years } \\
\text { (44.5\% males) }\end{array}$ & Cost-utility study & 2012 (USD) & - \\
\hline $\begin{array}{l}\text { Tamblyn } \\
2018[45]\end{array}$ & Canada (Quebec) & $\begin{array}{c}3592 \text { over } \\
18 \text { years } \\
\text { (44.7\% males) }\end{array}$ & $\begin{array}{l}\text { Single-blind cluster } \\
\text { randomized trial }\end{array}$ & 2017 (CAD) & $\begin{array}{c}\operatorname{lnt} \$ 1= \\
1.251 \mathrm{CAD}\end{array}$ \\
\hline $\begin{array}{l}\text { Vasudeva } \\
2016[46]\end{array}$ & USA & $35-74$ years & Cost-effectiveness study & 2015 (USD) & - \\
\hline $\begin{array}{l}\text { Wang G. } 2017 \\
\text { [47] }\end{array}$ & USA & $\begin{array}{c}40746 \text { over } \\
18 \text { years } \\
\text { (59.0\% males) }\end{array}$ & $\begin{array}{c}\text { Study based on data from } \\
\text { Medical Expenditure Panel } \\
\text { Survey }\end{array}$ & 2014 (USD) & - \\
\hline
\end{tabular}


Table I. Cont.

\begin{tabular}{|c|c|c|c|c|c|}
\hline Record & $\begin{array}{l}\text { Country } \\
\text { (region) }\end{array}$ & Population & Type of study & $\begin{array}{l}\text { Reference } \\
\text { year for costs } \\
\text { (currency) }\end{array}$ & $\begin{array}{l}\text { Purchasing } \\
\text { power } \\
\text { parity }\end{array}$ \\
\hline $\begin{array}{l}\text { Wang G., } \\
\text { Fang J. } 2014 \\
{[48]}\end{array}$ & USA & $\begin{array}{l}\text { Over } 25 \text { years, } \\
\text { mean age } \\
67.8 \text { years }\end{array}$ & $\begin{array}{l}\text { Study based on data from } \\
\text { National Hospital Discharge } \\
\text { Survey }\end{array}$ & 2008 (USD) & - \\
\hline $\begin{array}{l}\text { Wang G., } \\
\text { Zhang Z. } \\
2014[49]\end{array}$ & USA & $18-64$ years & $\begin{array}{l}\text { Study based on data from } \\
\text { MarketScan inpatient } \\
\text { database }\end{array}$ & 2008 (USD) & - \\
\hline $\begin{array}{l}\text { Wang Q. } 2014 \\
\text { [50] }\end{array}$ & China & $\begin{array}{c}1528 \text { over } \\
18 \text { years }\end{array}$ & $\begin{array}{l}\text { Study based on data } \\
\text { on medical payments }\end{array}$ & 2014 (USD) & - \\
\hline $\begin{array}{l}\text { Wang Z. } 2017 \\
\text { [51] }\end{array}$ & China & $\begin{array}{c}793 \text { aged } \\
18-70 \text { years, } \\
\text { mean age } \\
56.3 \text { years } \\
\text { (59.0\% males) }\end{array}$ & $\begin{array}{l}\text { Cost-effectiveness study, } \\
\text { randomized, open-label, } \\
\text { prospective clinical trial }\end{array}$ & 2016 (USD) & - \\
\hline $\begin{array}{l}\text { Weaver } 2015 \\
{[52]}\end{array}$ & Canada & $\begin{array}{l}608157 \text { mean } \\
\text { age } 62.3 \text { years } \\
(48.6 \% \text { males })\end{array}$ & $\begin{array}{c}\text { Study based on data from } \\
\text { Alberta Kidney Disease } \\
\text { Network }\end{array}$ & 2014 (CAD) & $\begin{array}{l}\text { Int\$ } 1= \\
1.23 \mathrm{CAD}\end{array}$ \\
\hline $\begin{array}{l}\text { Zhang } 2017 \\
{[53]}\end{array}$ & USA & Over 18 years & $\begin{array}{c}\text { Study based on data from } \\
\text { Medical Expenditure Panel } \\
\text { Survey }\end{array}$ & 2015 (USD) & - \\
\hline
\end{tabular}

Table II. Costs of arterial hypertension

\begin{tabular}{|c|c|c|c|}
\hline Record & $\begin{array}{l}\text { Total costs }(\mathrm{TC}) / \text { Total costs } \\
\text { per person }(\mathrm{TCpp}) \\
\text { Direct costs (DC)/Direct costs } \\
\text { per person (DCpp) } \\
\text { Indirect costs (IC)/Indirect } \\
\text { costs per person (ICpp) }\end{array}$ & $\begin{array}{c}\text { Hospital costs }(\mathrm{HC}) / \text { Hospital } \\
\text { costs per person ( } \mathrm{HCpp}) \\
\text { Hospital costs per admission } \\
(\mathrm{HCpa}) \\
\text { Drug costs (DrC)/Drug costs } \\
\text { per person (DrCpp) }\end{array}$ & $\begin{array}{c}\text { Pharmaceutical care costs } \\
\text { (PCC)/Pharmaceutical care } \\
\text { costs per person (PCCpp) } \\
\text { Out-of-pocket expenses } \\
\text { (OOPE) } \\
\text { Costs of stroke (COS)/Costs of } \\
\text { stroke per person (COSpp) }\end{array}$ \\
\hline $\begin{array}{l}\text { Alvarez-Sabin } \\
2016 \text { [15] }\end{array}$ & & & 41,575.54 Int\$ (COSpp) \\
\hline $\begin{array}{l}\text { Arredondo } \\
2015[16]\end{array}$ & $\begin{array}{c}6,880,020,349.00 \operatorname{Int} \text { (TC) } \\
3,261,937,129.00 \operatorname{Int} \text { (DC) } \\
3,618,083,220.00 \operatorname{Int} \$ \text { (IC) }\end{array}$ & $\begin{array}{l}358,812,888.00 \operatorname{lnt} \$(\mathrm{HC}) \\
456,671,390.00 \mathrm{Int} \$(\mathrm{DrC})\end{array}$ & $411,003,513.00$ Int\$ (COS) \\
\hline $\begin{array}{l}\text { Arredondo } \\
2016 \text { [17] }\end{array}$ & $\begin{array}{c}8,297,635.00 \text { Int\$ } \\
\text { (DC - Hidalgo)/796.17 Int\$ } \\
\text { (DCpp - Hidalgo) } \\
18,413,820.00 \text { Int\$ } \\
\text { (DC - Jalisco)/590.77 Int\$ } \\
\text { (DCpp - Jalisco) } \\
\text { 10,007,786.00 Int\$ } \\
\text { (DC - Morelos)/625.10 Int\$ } \\
\text { (DCpp - Morelos) } \\
\text { 14,769,269.00 Int\$ } \\
\text { (DC - Sinaloa)/470.27 Int\$ } \\
\text { (DCpp - Sinaloa) } \\
5,485,622.00 \text { Int\$ } \\
\text { (DC - Yucatán)/579.57 Int\$ } \\
\text { (DCpp - Yucatán) }\end{array}$ & & \\
\hline $\begin{array}{l}\text { Bernard } 2014 \\
{[18]}\end{array}$ & & & 174.00 Int\$ (OOPE) \\
\hline $\begin{array}{l}\text { Bigdeli } 2016 \\
\text { [19] }\end{array}$ & 399.00 Int\$ (DCpp) & 350.00 Int\$ (DrCpp) & \\
\hline $\begin{array}{l}\text { Boan } 2014 \\
{[20]}\end{array}$ & & & $2,770,000,000.00 \operatorname{lnt} \$(\mathrm{COS})$ \\
\hline $\begin{array}{l}\text { Boubouch- } \\
\text { airopoulou } \\
2014[21]\end{array}$ & 2,411.62 Int\$ (DCpp) & 405.24 Int\$ (DrCpp) & \\
\hline
\end{tabular}


Table II. Cont.

\begin{tabular}{|c|c|c|c|}
\hline Record & $\begin{array}{l}\text { Total costs (TC)/Total costs } \\
\text { per person (TCpp) } \\
\text { Direct costs (DC)/Direct costs } \\
\text { per person (DCpp) } \\
\text { Indirect costs (IC)/Indirect } \\
\text { costs per person (ICpp) }\end{array}$ & $\begin{array}{l}\text { Hospital costs (HC)/ Hospital } \\
\text { costs per person (HCpp) } \\
\text { Hospital costs per admission } \\
\text { (HCpa) } \\
\text { Drug costs (DrC)/Drug costs } \\
\text { per person (DrCpp) }\end{array}$ & $\begin{array}{l}\text { Pharmaceutical care costs } \\
\text { (PCC)/Pharmaceutical care } \\
\text { costs per person (PCCpp) } \\
\text { Out-of-pocket expenses } \\
\text { (OOPE) } \\
\text { Costs of stroke (COS)/Costs of } \\
\text { stroke per person (COSpp) }\end{array}$ \\
\hline $\begin{array}{l}\text { Bueno } 2017 \\
{[22]}\end{array}$ & & 30.23 Int\$ (DrCpp) & \\
\hline $\begin{array}{l}\text { Cazarim } 2018 \\
{[23]}\end{array}$ & & & $\begin{array}{c}23,350.38 \operatorname{lnt} \$(P C C) / 224.52 \\
\text { Int\$ (PCCpp) }\end{array}$ \\
\hline $\begin{array}{l}\text { Finkelstein } \\
2014[24]\end{array}$ & $\begin{array}{c}\text { 1,320,000,000.00 Int\$ } \\
\text { (TC)/70.00 Int\$ (TCpp) } \\
\text { 540,000,000.00 Int\$ } \\
\text { (DC)/30.00 Int\$ (DCpp) } \\
\text { 780,000,000.00 Int\$ (IC)/50.00 } \\
\text { Int\$ (ICpp) }\end{array}$ & & $\begin{array}{c}290,000,000.00 \mathrm{Int} \$ \\
(\operatorname{CoS}) / 380.00 \mathrm{Int} \$(\mathrm{COSpp})\end{array}$ \\
\hline $\begin{array}{l}\text { Gilmer } 2014 \\
{[25]}\end{array}$ & 1,972.00 Int\$ (DCpp) & & \\
\hline $\begin{array}{l}\text { Huang } 2016 \\
{[26]}\end{array}$ & 193.32 Int\$ (DCpp) & 2,967.90 Int\$ (HCpa) & 45.68 Int\$ (OOPE) \\
\hline $\begin{array}{l}\text { Kawalec } 2015 \\
{[27]}\end{array}$ & & & $9,439.26$ Int\$ (OOPE) \\
\hline $\begin{array}{l}\text { Kirkland } 2018 \\
{[28]}\end{array}$ & 9,089.00 Int\$ (DCpp) & $\begin{array}{l}2,731.00 \operatorname{lnt} \$(\mathrm{HCpp}) \\
2,371.00 \operatorname{lnt} \$(\mathrm{DrCpp})\end{array}$ & \\
\hline $\begin{array}{l}\text { Lekoubou } \\
2018[29]\end{array}$ & $\begin{array}{c}316,000,000,000.00 \mathrm{Int} \$(\mathrm{TC}) \\
193,000,000,000.00 \mathrm{Int} \$(\mathrm{DC}) \\
123,000,000,000.00 \mathrm{Int} \text { (IC) }\end{array}$ & & $\begin{array}{c}98,300,000,000.00 \operatorname{Int} \$ \\
(\operatorname{COS}) / 17,165.00 \operatorname{lnt} \$(\operatorname{CoSpp})\end{array}$ \\
\hline Liu 2016 [30] & & 1,936.23 Int\$ (HCpa) & \\
\hline $\begin{array}{l}\text { Marra } 2017 \\
{[31]}\end{array}$ & & & $\begin{array}{c}209,658.38 \text { Int\$ (PCC) } \\
64,093.83 \text { Int\$ (COSpp) }\end{array}$ \\
\hline $\begin{array}{l}\text { Milan } 2017 \\
{[32]}\end{array}$ & & & 28.78 Int\$ (OOPE) \\
\hline $\begin{array}{l}\text { Mitchell- } \\
\text { Fearon } 2017 \\
{[10]}\end{array}$ & $\begin{array}{c}204,676,723.54 \text { Int\$ } \\
\text { (TC)/1,190.28 Int\$ (TCpp) } \\
116,173,000.90 \text { Int\$ } \\
\text { (DC)/675.59 Int\$ (DCpp) } \\
88,503,722.64 \text { Int\$ (IC)/514.68 } \\
\text { Int\$ (ICpp) }\end{array}$ & $\begin{array}{c}10,851,082.11 \text { Int\$ (HC)/3.10 } \\
\text { Int\$ (HCpp) } \\
\text { 37,038,595.58 Int\$ } \\
(\text { DrC)/215.39 Int\$ (DrCpp) }\end{array}$ & $3,000.00 \operatorname{lnt} \$$ (OOPE) \\
\hline $\begin{array}{l}\text { Moise } 2016 \\
{[33]}\end{array}$ & & 11,994.00 Int\$ (HCpp) & $26,000.00 \operatorname{lnt} \$(\mathrm{COSpp})$ \\
\hline $\begin{array}{l}\text { Murphy } 2016 \\
{[34]}\end{array}$ & & & 12.70 Int\$ (OOPE) \\
\hline $\begin{array}{l}\text { Mutowo } 2016 \\
{[35]}\end{array}$ & & 914.00 Int\$ (HCpa) & \\
\hline $\begin{array}{l}\text { Nguyen } 2014 \\
{[36]}\end{array}$ & & 64.95 Int\$ (HCpa) & \\
\hline $\begin{array}{l}\text { Paczkowska } \\
2014[37]\end{array}$ & 116.08 Int\$ (DCpp) & $\begin{array}{l}159.82 \text { Int\$ (HCpp) } \\
68.84 \text { Int\$ (DrCpp) }\end{array}$ & \\
\hline $\begin{array}{l}\text { Park } 2017 \\
{[38]}\end{array}$ & 9,841.00 Int\$ (DCpp) & & $9,555.00 \operatorname{lnt} \$$ (COSpp) \\
\hline $\begin{array}{l}\text { Ricci } 2017 \\
\text { [39] }\end{array}$ & $3,666,930,171.28 \operatorname{lnt} \$(D C)$ & & \\
\hline $\begin{array}{l}\text { Ritchey } 2016 \\
{[40]}\end{array}$ & & $28,810,000,000.00 \operatorname{lnt} \$(\mathrm{DrC})$ & 359.00 IntS (OOPE) \\
\hline $\begin{array}{l}\text { Shireman } \\
2016[41]\end{array}$ & & & 104.80 Int\$ (PCCpp) \\
\hline
\end{tabular}


Table II. Cont.

\begin{tabular}{|c|c|c|c|}
\hline Record & $\begin{array}{c}\text { Total costs }(\mathrm{TC}) / \text { Total costs } \\
\text { per person }(\mathrm{TCpp}) \\
\text { Direct costs }(\mathrm{DC}) / \mathrm{Direct} \text { costs } \\
\text { per person }(\mathrm{DCpp}) \\
\text { Indirect costs }(\mathrm{IC}) / \text { Indirect } \\
\text { costs per person (ICpp) }\end{array}$ & $\begin{array}{c}\text { Hospital costs }(\mathrm{HC}) / \text { Hospital } \\
\text { costs per person }(\mathrm{HCpp}) \\
\text { Hospital costs per admission } \\
(\mathrm{HCpa}) \\
\text { Drug costs }(\mathrm{DrC}) / \mathrm{Drug} \text { costs } \\
\text { per person (DrCpp) }\end{array}$ & $\begin{array}{c}\text { Pharmaceutical care costs } \\
\text { (PCC)/Pharmaceutical care } \\
\text { costs per person (PCCpp) } \\
\text { Out-of-pocket expenses } \\
\text { (OOPE) } \\
\text { Costs of stroke (COS)/Costs of } \\
\text { stroke per person (COSpp) }\end{array}$ \\
\hline $\begin{array}{l}\text { Specogna } \\
2017[42]\end{array}$ & & & $12,856.72 \operatorname{lnt}$ (COSpp) \\
\hline $\begin{array}{l}\text { Stafylas } 2015 \\
{[43]}\end{array}$ & & $\begin{array}{c}4,154.65 \operatorname{Int} \$ \text { (HCpp) } \\
10,877.88 \operatorname{Int} \$ \text { (DrCpp) }\end{array}$ & \\
\hline $\begin{array}{l}\text { Tajeu } 2017 \\
{[44]}\end{array}$ & & 189.48 Int\$ (DrCpp) & 69,642.00 Int\$ (COSpp) \\
\hline $\begin{array}{l}\text { Tamblyn } \\
2018[45]\end{array}$ & & 436.77 Int\$ (DrCpp) & 209.03 Int\$ (OOPE) \\
\hline $\begin{array}{l}\text { Vasudeva } \\
2016[46]\end{array}$ & & 12,000.00 Int\$ (HCpp) & $26,000.00$ IntS (COSpp) \\
\hline $\begin{array}{l}\text { Wang G. } 2017 \\
{[47]}\end{array}$ & 8,854.00 Int\$ (DCpp) & & \\
\hline $\begin{array}{l}\text { Wang G., } \\
\text { Fang J. } 2014 \\
\text { [48] }\end{array}$ & & $\begin{array}{c}113,390,000,000.00 \mathrm{Int} \$(\mathrm{HC}) \\
8,480.00 \mathrm{Int} \$(\mathrm{HCpa})\end{array}$ & \\
\hline $\begin{array}{l}\text { Wang G., } \\
\text { Zhang Z. } \\
2014 \text { [49] }\end{array}$ & & & $23,256.00 \operatorname{lntS}(\mathrm{COSpp})$ \\
\hline $\begin{array}{l}\text { Wang Q. } 2014 \\
\text { [50] }\end{array}$ & & & 93.50 Int\$ (OOPE) \\
\hline $\begin{array}{l}\text { Wang Z. } 2017 \\
\text { [51] }\end{array}$ & & 63.40 Int\$ (DrCpp) & \\
\hline $\begin{array}{l}\text { Weaver } 2015 \\
{[52]}\end{array}$ & $\begin{array}{c}11,300,813,008.13 \mathrm{Int} \$ \\
(\mathrm{DC}) / 1,903.25 \mathrm{Int} \$ \text { (DCpp) }\end{array}$ & & \\
\hline $\begin{array}{l}\text { Zhang } 2017 \\
{[53]}\end{array}$ & 1494.00 USD Int\$ (DCpp) & & \\
\hline
\end{tabular}

USD, and indirect costs at 4,600,000,000 USD (with the last amount seeming grossly underestimated). The current review estimates HT costs in the USA as follows: total costs - 316,000,000,000 Int\$, direct costs - 193,000,000,000 Int\$, indirect costs - 123,000,000,000 Int\$. The USD-Int\$ exchange rate is always $1: 1$. The data presented in this review seem to be more reliable. According to a recent study from Australia, indirect HT costs amount to $137,200,000,000$ AUD (Hird 2019) [56].

Goetzel estimated direct costs per capita in the USA at 392 USD (Goetzel 2004) [57], while Burnier suggested a much wider range between 4,871 and 11,238 USD, depending on patient compliance as regards medication (Burnier 2019) [58]. Owing to the generally high prices in the USA, direct costs per person in the region of several thousand USD appear to be more realistic than amounts in the region of a few hundred USD. The high costs in the USA are also confirmed by the current review, where direct costs per person are estimated at
6,250.00 IntS in the USA, and $1,903.25$ IntS in Canada.

The British National Health Service spends 250,000,000 GBP (pound sterling) annually only on angiotensin II receptor blockers used as antihypertensive medications (Grosso 2011) [59]. In 2010 in Malaysia, the annual cost of HT drugs was estimated to be 6,273.70 MYR per patient [60]. There are also studies reporting pharmacotherapy costs in dollars per quality-adjusted life-year (QALYS) gained. They were not included in this review due to difficulty comparing financial costs with costs per QALY. For example, in the Republic of South Africa drug costs in dollars per QALYs gained ranged from 700 to 11,000 USD, depending on the number of cardiovascular risk factors present (Gaziano 2005) [61]. In the USA, drug costs per QALY are as follows: angiotensin-converting-enzyme inhibitors - 34,244 USD, calcium channel blockers - 13,016 USD, $\beta$-blockers - from 1,498 to 18,137 USD (Park 2017) [62]. In the USA, drug costs per hos- 


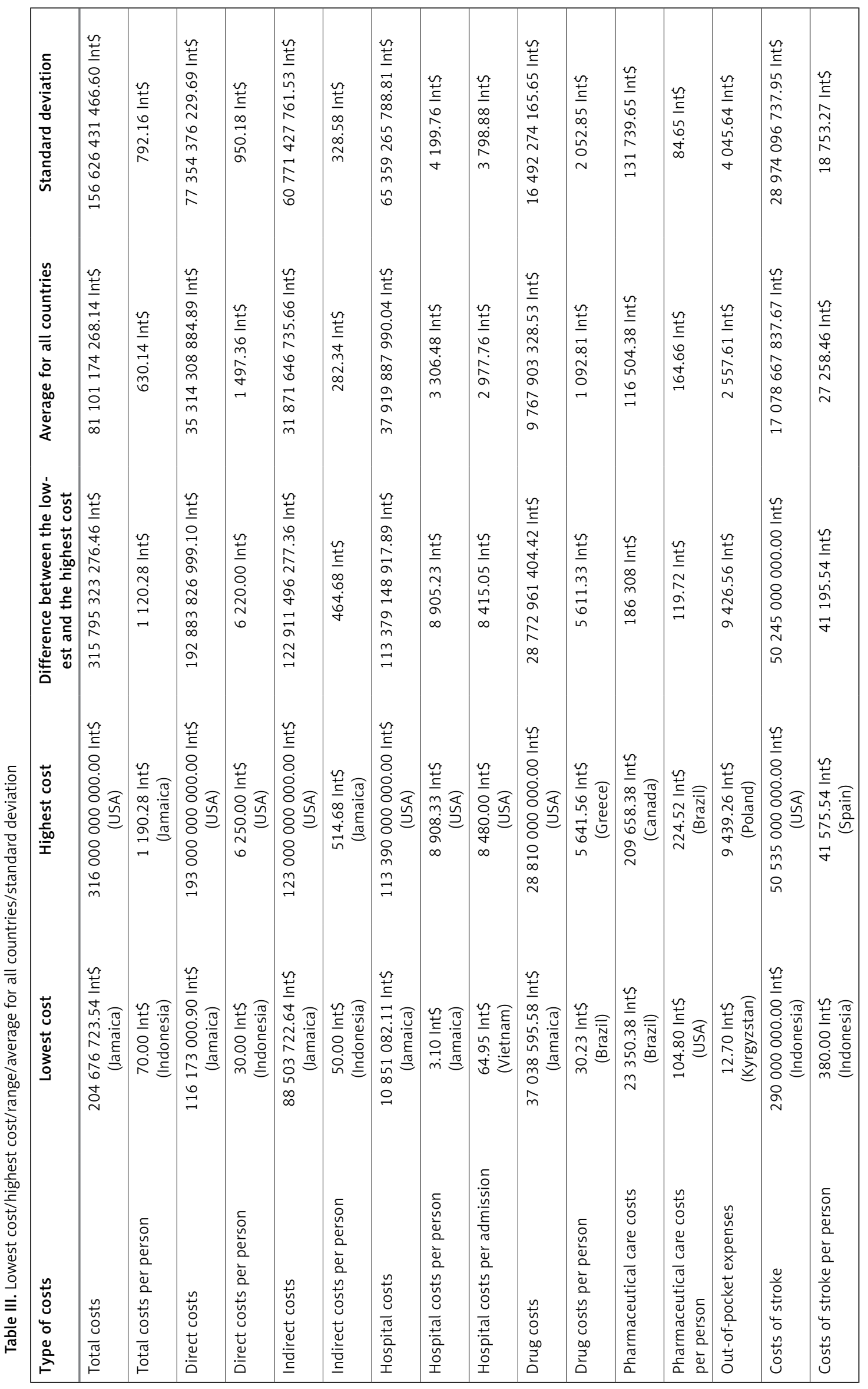


Table IV. Proportions of direct and indirect costs in total costs

\begin{tabular}{|lcc|}
\hline Country & Direct costs (\%) & Indirect costs (\%) \\
\hline Indonesia & 41 & 59 \\
\hline Jamaica & 57 & 43 \\
\hline Mexico & 47 & 53 \\
\hline USA & 61 & 39 \\
\hline Average & 51.5 & 48.5 \\
\hline
\end{tabular}

pitalized person treated with sacubitril/valsartan amount to 248 USD, while costs per hospitalized person treated with angiotensin-converting-enzyme inhibitors or angiotensin II receptor blockers amount to 1,122 USD (Albert 2019) [63]. Thus, the sacubitril/valsartan treatment seems the most cost-effective pharmacological treatment. This review estimates the mean drug costs for all the analyzed countries at 9,767,903,328.53 Int\$ in total and at 1,092.81 Int\$ per person.

Among the studies exploring costs of stroke, the most comprehensive is that by Mullins (2004), estimating post-stroke hospitalization costs per person to be 23,109 USD in Canada, 16,415 USD in the USA, 7,703 USD in Spain, 5,003 USD in France, 4,980 USD in Italy, 4,567 USD in Germany, 2,639 USD in Australia and 2,607 USD in the UK. According to the same study, post-stroke inpatient care costs per person amount to 31,694 USD in the USA, 14,188 USD in France, 12,744 USD in Australia, 7,188 USD in Germany, 3,094 USD in Spain, and 606 USD in the UK (Mullins 2004) [64]. According to a different estimate, stroke costs per person in Canada equal 110,471 CAD (Canadian dollar) (Anis 2006) [65]. In Finland, they were estimated to reach 1,600,000,000 USD annually, while costs per person for a year after a stroke ranged from 29,580 to 42,570 USD depending on the kind of stroke, with higher costs generated by hemorrhagic strokes related to HT (Meretoja 2007) [66]. In Sweden, stroke costs per person for a year after a stroke ranged from 15,970 to 39,254 USD depending on stroke severity (Claesson 2000) [62]. According to European research, hospital costs per admission caused by strokes ranged from 466 USD in Latvia to 8,512 USD in Denmark (Grieve 2001) [67]. In the UK, post-stroke rehabilitation costs ranged from 326 to 19,901 GBP per person (Luengo-Fernandez 2006) [68]. In Canada, post-hospital care for stroke patients after early supported discharge was estimated to cost 7,748 USD per person, while traditional hospital care was estimated to cost 11,065 USD per person (Teng 2003) [69]. To compare, this review estimates mean stroke costs for all the analyzed countries at 17,078,667,837.67 Int\$ in total, and 27,258.46 Int\$ per person.
Table V. Cost structure within direct costs

\begin{tabular}{|lccc|}
\hline Country & $\begin{array}{c}\text { Hospital } \\
\text { costs (\%) }\end{array}$ & $\begin{array}{c}\text { Drug costs } \\
(\%)\end{array}$ & $\begin{array}{c}\text { Other costs } \\
(\%)\end{array}$ \\
\hline Jamaica & 9 & 32 & 59 \\
\hline Mexico & 11 & 14 & 75 \\
\hline USA & 59 & 15 & 26 \\
\hline Average & 26.33 & 20.33 & 53.33 \\
\hline
\end{tabular}

Other interesting conclusions can be reached when comparing selected indices of the social and economic situation of the studied countries with HT costs reported for these countries (Tables VI and VII). Full consistency was observed between the ranking of the countries according to HT costs and their ranking according to health expenditure as a percentage of gross domestic product (GDP). Similar (but not always full) consistency can be seen for countries ranked by HT costs and by other indices such as Human Development Index (HDI), GDP per capita (Int\$), health expenditure per capita (Int\$), domestic general government health expenditure per capita (Int\$), out-of-pocket expenses (Int\$), coverage of essential health services, the number of physicians per 1,000 population, the number of deaths caused by NCDs (non-communicable diseases), the percentage of the population aged 18 years or older with BMI (body mass index) $>30$, the number of liters of pure alcohol consumed per person aged 15 years or older, and the age-standardized prevalence of insufficient physical activity in the population aged 18 years or older. The analyses of the convergence between country rankings based on these indices and country rankings based on HT costs showed one difference in each case, i.e. one country holding a different rank in one of the paired rankings. The country was usually Jamaica, whose position in cost rankings was lower than the position suggested by rankings of the particular socioeconomic indices (Tables VI and VII).

The results suggest that the indices mentioned above may influence HT costs. The two questions for future research are, whether there is a causal relationship between the indices and the costs, and how strong the influence is. Such analyses should involve a bigger, representative number of countries, with a bigger number of sources providing reliable data.

The authors admit that the present review has a number of limitations. The studies analyzed in the review cover only 15 countries, thus excluding almost $65 \%$ of the global population and such populous countries as India. The review data came from only 3 sources (PubMed, Cochrane, and Goo- 


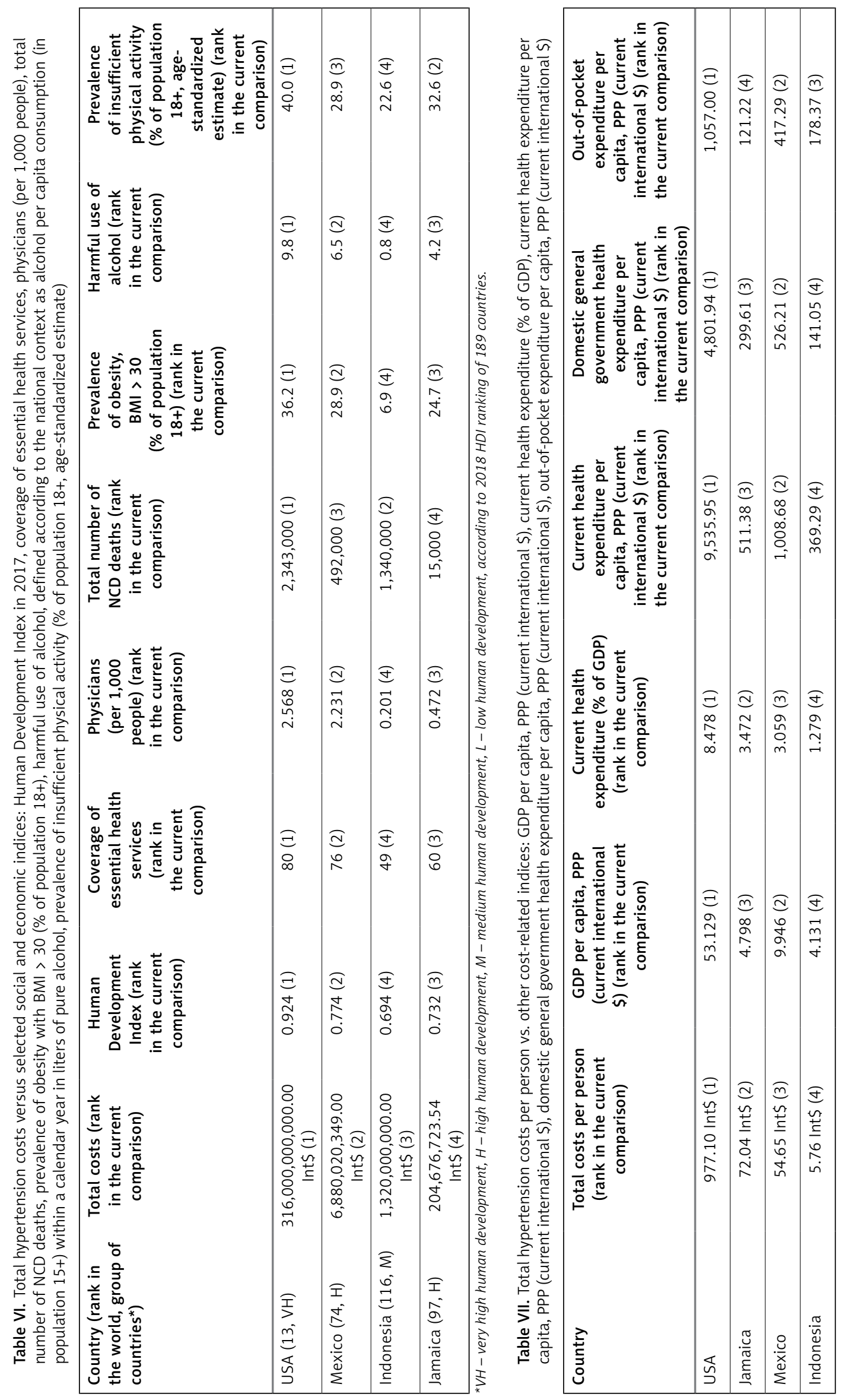


gle Scholar), and numerous relevant studies were presumably not listed there. As the search algorithm of Google Scholar is not known, it could only be treated as an additional source. The current review included few studies describing HT costs in European countries. Some cost categories, e.g. prevention costs, were missing; other categories were presented in such a way that making comparisons of similar data from different sources turned out to be impossible. Moreover, the information was often fragmentary - no country had a complete set of data presenting all cost categories and methods of calculating them. Consequently, costs in some categories may be skewed, i.e. over- or under-estimated, when the data for these categories included countries with particularly high or low healthcare expenditures. It should also be noted that the HT definition used for the purposes of this review is blood pressure exceeding 140/90 mm Hg, while the 2017 ACC Guideline for Hypertension sets the limit at $130 / 80 \mathrm{~mm} \mathrm{Hg}$ [2]. The more lenient limit was adopted here because the cost data included in the review come partly from before the year 2017.

In conclusion, the current review collated data from countries whose populations altogether constitute over $35 \%$ of the global population. They include some of the most populous countries in the world: China, the USA, Indonesia, Brazil. Although the databases selected for the review provided numerous records whose titles suggested relevance to HT costs, the records actually did not pertain to HT costs or sometimes even to HT itself. The 40 articles finally included in the review turned out to be very heterogeneous with respect to the cost categories and cost sources discussed, which made comparing the data difficult. The quality of data was mostly low or moderate and fitted in the 2 middle quality categories out of 4 (very low, low, moderate, high). Additional analyses showed interesting relations between direct and indirect costs, as well as within the structure of direct costs between hospital costs, drug costs and other costs. A comparison of country rankings according to costs and according to selected economic and social indices presented an increase of costs parallel to an increase of the indices.

When compared with other studies, this review usually listed higher HT costs. This may be due to the fact that the review included articles from the last 5 years, while the literature for discussion was selected from a slightly longer period. This can testify to the fact that healthcare costs, including HT costs, are growing year by year. The trend is corroborated by the World Bank data [70]. One can expect that HT costs will be an increasingly heavy burden on society, so continuous research pertaining to this problem is necessary. To achieve this, it is necessary to develop official and uniform cost terminology and definitions and to introduce uniform standards for calculating healthcare costs to make cost comparisons easier. The improvement of data collection and publication methods would also greatly facilitate the process. All of the above measures would enable better monitoring and analysis of cost types and cost centers, which would facilitate making more informed decisions regarding fund allocation in health policies.

\section{Conflict of interest}

The authors declare no conflict of interest.

\section{References}

1. Dai S, Huang B, Zou Y, Liu Y. Associations of dipping and non-dipping hypertension with cardiovascular diseases in patients with dyslipidemia. Arch Med Sci 2019; 15: 337-42.

2. Whelton PK, Carey RM, Aronow WS, et al. 2017 ACC/ AHA/AAPA/ABC/ACPM/AGS/APhA/ASH/ASPC/NMA/ PCNA Guideline for the Prevention, Detection, Evaluation, and Management of High Blood Pressure in Adults. A Report of the American College of Cardiology/American Heart Association Task Force on Clinical Practice Guidelines. Hypertension 2018; 71: 1269-324.

3. Bielecka-Dabrowa A, Aronow WS, Rysz J, Banach M. The rise and fall of hypertension: lessons learned from Eastern Europe. Curr Cardiovasc Risk Rep 2011; 5: 174-9.

4. Du Z, Bian W, Wu S, et al. Effects of blood pressure goals on cardiovascular outcomes in hypertensive patients. Arch Med Sci 2018; 15: 1381-7.

5. Chruściel P, Stemplewska P, Stemplewski A, et al. Associations between the lipid profile and the development of hypertension in young individuals - the preliminary study. Arch Med Sci doi:10.5114/aoms.2019.86197.

6. Miazgowski T, Kopec J, Widecka K, Miazgowski B, Kaczmarkiewicz A. Epidemiology of hypertensive heart disease in Poland: findings from the Global Burden of Disease Study 2016. Arch Med Sci doi:10.5114/ aoms.2019.85222.

7. Irzmanski R, Barylski M, Banach $M$, et al. The concentration of atrial and brain natriuretic peptide in patients with idiopathic hypertension. Med Sci Monit 2007; 13: 449-56.

8. Bielecka-Dabrowa A, Gluba-Brzózka A, Michalska-Kasiczak M, Misztal M, Rysz J, Banach M. The multi-biomarker approach for heart failure in patients with hypertension. Int J Mol Sci 2015; 16: 10715-33.

9. Pistoia F, Sacco S, Degan D, Tiseo C, Ornello R, Carolei A. Hypertension and stroke: epidemiological aspects and clinical evaluation. High Blood Press Cardiovasc Prev 2016; 23: 9-18.

10. Mitchell-Fearon K, McKoy-Davis J, Willie-Tyndale D, Abdulkadri AO, Eldemire-Shearer D. The economic burden of hypertension among older persons: lessons from a developing nation. J Public Health Dev Ctries 2017; 3: 347-57.

11. Turin TC, Okamura T, Afzal AR, et al. Hypertension and lifetime risk of stroke. J Hypertens 2016; 34: 116-22.

12. Hutton B, Salanti G, Caldwell DM, Chaimani A, Schmid CH. The PRISMA extension statement for reporting of systematic reviews incorporating network meta-analyses 
of health care interventions: checklist and explanations. Ann Intern Med 2015; 162: 777-84.

13. Downs $\mathrm{H}$, Black N. The feasibility of creating a checklist for the assessment of the methodological quality both of randomised and non-randomised studies of health care interventions. J Epidemiol Community Health 1998; 52: 377-84.

14. The World Bank. PPP conversion factor. https://data worldbank.org/indicator/PA.NUS.PPP?end $=2017 \&$ start=2017\&view=map (access: 1 July 2019).

15. Alvarez-Sabın J, Quintana M, Masjuan J, et al. Economic impact of patients admitted to stroke units in Spain. Eur J Health Econ 2017; 18: 449-58.

16. Arredondo A, Cuadra SM, Duarte MB. Challenges of the epidemiological and economic burdens associated with hypertension in middle income countries: evidence from Mexico. BMC Public Health 2015; 15: 1106.

17. Arredondo A, Orozco E, Alcalde-Rabanal J, Navarro J, Azar A. Challenges on the epidemiological and economic burden of diabetes and hypertension in Mexico. Rev Saude Publica 2018; 52: 23.

18. Bernard DM, Johansson P, Fang Z. Out-of-pocket healthcare expenditure burdens among nonelderly adults with hypertension. Am J Manag Care 2014; 20: 406-13.

19. Bigdeli M, Jacobs B, Men CR, Nilsen K, Van Damme W, Dujardin B. Access to treatment for diabetes and hypertension in rural cambodia: performance of existing social health protection schemes. PLoS One 2016; 11: e0146147.

20. Boan AD, Feng WW, Ovbiagele B. Persistent racial disparity in stroke hospitalization and economic impact in young adults in the buckle of stroke belt. Stroke 2014 45: 1932-8.

21. Boubouchairopoulou N, Karpettas N, Athanasakis K. Cost estimation of hypertension management based on home blood pressure monitoring alone or combined office and ambulatory blood pressure measurements. J Am Soc Hypertens 2014; 8: 732-8.

22. Bueno DR, de Fátima Nunes Marucci M, Gobbo LA, de Almeida-Roediger $M$, de Oliveira Duarte YA, Lebrão ML. Expenditures of medicine use in hypertensive/diabetic elderly and physical activity and engagement in walking: cross secctional analysis of SABE Survey. BMC Geriatrics 2017; 17: 70.

23. Cazarim MS, Pereira LRL. Cost-effectiveness analysis of pharmaceutical care for hypertensive patients from the perspective of the public health system in Brazil. PLoS One 2018; 13: e0193567.

24. Finkelstein EA, Chay J, Bajpai S. The economic burden of self-reported and undiagnosed cardiovascular diseases and diabetes on indonesian households. PLoS One 2014; 9: e99572.

25. Gilmer TP, O'Connor PJ, Sinaiko AR. Impact of hypertension on health care costs among children. Am J Manag Care 2014; 20: 622-8.

26. Huang Y, Liu Y, Yang X, Li J, Fang P. Global budget payment system helps to reduce outpatient medical expenditure of hypertension in China. SpringerPlus 2016; 5: 1877.

27. Kawalec P, Holko P, Stawowczyk E, Borowiec $Ł$, Filipiak KJ. Economic evaluation of single-pill combination of indapamide and amlodipine in the treatment of arterial hypertension in the Polish setting. Kardiol Pol 2015; 73: 768-80.

28. Kirkland EB, Heincelman M, Bishu KG, et al. Trends in healthcare expenditures among US adults with hypertension: national estimates, 2003-2014. J Am Heart Assoc 2018; 7: e008731.

29. Lekoubou A, Bishu KG, Ovbiagele B. Nationwide healthcare expenditures among hypertensive individuals with stroke: 2003-2014. J Stroke Cerebrovasc Dis 2018; 27 : 1760-9.

30. Liu X, Sun $X$, Zhao $Y$, Meng $O$. Financial protection of rural health insurance for patients with hypertension and diabetes: repeated cross-sectional surveys in rural China. BMC Health Serv Res 2016; 16: 481.

31. Marra C, Johnston K, Santschi V, Tsuyuki RT. Cost-effectiveness of pharmacist care for managing hypertension in Canada. Can Pharm J 2017; 150: 184-97.

32. Milan R, Vasiliadis HM, Gontijo Guerra S, Berbiche D. Out-of-pocket costs and adherence to antihypertensive agents among older adults covered by the public drug insurance plan in Quebec. Patient Prefer Adherence 2017; 11: 1513-22.

33. Moise N, Huang C, Rodgers A, et al. Comparative cost-effectiveness of conservative or intensive blood pressure treatment guidelines in adults aged 35-74 years: the CVD Policy Model. Hypertension 2016; 68: 88-96.

34. Murphy A, Jakab M, McKee M, Richardson E. Persistent low adherence to hypertension treatment in Kyrgyzstan: how can we understand the role of drug affordability? Health Policy Plan 2016; 31: 1384-90.

35. Mutowo MP, Lorgelly PK, Laxy M, Renzaho AMN, Mangwiro JC, Owen AJ. The hospitalization costs of diabetes and hypertension complications in Zimbabwe: estimations and correlations. J Diabetes Res 2016; 2016 9754230.

36. Nguyen TP, Nguyen TB, Nguyen TT, et al. Direct costs of hypertensive patients admitted to hospital in Vietnam - a bottom-up micro-costing analysis. BMC Health Serv Res 2014; 14: 514.

37. Paczkowska A, Koligat D, Nowakowska E, Hoffmann K, Bryl W. Analysis of direct costs of hypertension treatment among adolescents in Poland. Acta Polon Pharmaceut Drug Res 2014; 71: 197-203.

38. Park C, Fang J, Hawkins NA, Wang G. Comorbidity status and annual total medical expenditures in U.S. hypertensive adults. Am J Prev Med 2017; 53 (6 Suppl 2): 172-81.

39. Ricci G, Pirillo I, Tomassoni D, Sirignano A, Grappasonni I. Metabolic syndrome, hypertension, and nervous system injury: epidemiological correlates. Clin Exp Hypertens 2017; 39: 8-16.

40. Ritchey M, Tsipas S, Loustalot F, Wozniak G. Use of pharmacy sales data to assess changes in prescriptionand payment-related factors that promote adherence to medications commonly used to treat hypertension, 2009 and 2014. PLoS One 2016; 11: e0159366.

41. Shireman TI, Svarstad BL. Cost-effectiveness of Wisconsin TEAM model for improving adherence and hypertension control in black patients. J Am Pharm Assoc 2016; 56: 389-96.

42. Specogna AV, Turin TC, Patten SB, Hill MD. Hospital treatment costs and length of stay associated with hypertension and multimorbidity after hemorrhagic stroke. BMC Neurol 2017; 17: 158.

43. Stafylas P, Kourlaba G, Hatzikou M, Georgiopoulos D, Sarafidis P, Maniadakis N. Economic evaluation of a single-pill triple antihypertensive therapy with valsartan, amlodipine, and hydrochlorothiazide against its dual components. Cost Eff Resour Alloc 2015; 13: 10.

44. Tajeu GS, Mennemeyer S, Menachemi N, Weech-Maldonado R, Kilgore M. Cost-effectiveness of antihypertensive medication: exploring race and sex differences using data from the easons for geographic and racial differences in stroke study. Med Care 2017; 55: 552-60.

45. Tamblyn R, Winslade N, Qian CJ, Moraga T, Huang A. What is in your wallet? A cluster randomized trial of the 
effects of showing comparative patient out-of-pocket costs on primary care prescribing for uncomplicated hypertension. Implement Sci 2018; 13: 7.

46. Vasudeva E, Moise N, Huang C, et al. Comparative cost-effectiveness of hypertension treatment in nonHispanic blacks and whites according to 2014 guidelines: a modeling study. Am J Hypertens 2016; 29: 1195205.

47. Wang G, Zhou X, Zhuo X, Zhang P. Annual total medical expenditures associated with hypertension by diabetes status in U.S. adults. Am J Prev Med 2017; 53 (6 Suppl 2): 182-9.

48. Wang G, Fang J, Ayala C. Hypertension-associated hospitalizations and costs in the United States, 1979-2006. Blood Press 2014; 23: 126-33.

49. Wang G, Zhang Z, Ayala C, Dunet DO, Fang J, George MG. Costs of hospitalization for stroke patients aged 18-64 years in the United States. J Stroke Cerebrovasc Dis 2014; 23: 861-8.

50. Wang Q, Liu H, Lu ZX, Luo Q, Liu JA. Role of the new rural cooperative medical system in alleviating catastrophic medical payments for hypertension, stroke and coronary heart disease in poor rural areas of China. BMC Public Health 2014; 14: 907.

51. Wang Z, Chen Z, Wang X. Cost-effectiveness of nitrendipine and hydrochlorothiazide or metoprolol to treat hypertension in rural community health centers in China. J Hypertens 2017; 35: 886-92.

52. Weaver CG, Clement FM, Campbell NRC, et al. Healthcare costs attributable to hypertension Canadian population-based cohort study. Hypertension 2015; 66: 502-8.

53. Zhang D, Wang G, Zhang P, Fang J, Ayala C. Medical expenditures associated with hypertension in the U.S., 2000-2013. Am J Prev Med 2017; 53 (6 Suppl 2): 164-71.

54. Mullins CD, Sikirica M, Seneviratne V, Ahn J, Akhras KS. Comparisons of hypertension-related costs from multinational clinical studies. Pharmacoeconomics 2004; 22: 1001-14.

55. Benjamin EJ, Muntner P, Alonso A, et al. Heart disease and stroke statistics - 2019 update. A Report from the American Heart Association. Circulation 2019; 139: 52-6.

56. Hird TR, Zomer E, Owen AJ, Magliano DJ, Liew D. Productivity burden of hypertension in Australia. Hypertension 2019; 73: 777-84

57. Goetzel RZ, Long SR, Ozminkowski RJ, Hawkins K, Wang S, Lynch W. Health, absence, disability, and presenteeism cost estimates of certain physical and mental health conditions affecting U.S. employers. J Occup Environ Med 2004; 46: 398-412.

58. Burnier M, Egan BM. Adherence in hypertension. A review of prevalence, risk factors, impact, and management. Circ Res 2019; 124: 1124-40.

59. Grosso AM, Bodalia PN, MacAllister RJ, Hingorani AD, Moon JC, Scott MA. Comparative clinical- and cost-effectiveness of candesartan and losartan in the management of hypertension and heart failure: a systematic review, meta- and cost-utility analysis. Int J Clin Pract 2011; 65: 253-63.

60. Mohd-Tahir NA, Li SC. Budget impact analysis of increasing prescription of renin-angiotensin system inhibitors drugs to standard anti-hypertensive treatments in patients with diabetes and hypertension in a hypothetical cohort of Malaysian population. PLoS One 2019; 14: e0212832.
61. Gaziano TA, Steyn K, Cohen DJ, Weinstein MC, Opie LH. Cost-effectiveness analysis of hypertension guidelines in South Africa. Absolute risk versus blood pressure level. Circulation 2005; 112: 3569-76.

62. Park C, Wang G, Durthaler JM, Fang J. Cost-effectiveness analyses of antihypertensive medicines: a systematic review. Am J Prev Med 2017; 53 (6 Suppl 2): 131-42.

63. Albert NM, Swindle JP, Buysman EK, Chang C. Lower hospitalization and healthcare costs with sacubitril/valsartan versus angiotensin-converting enzyme inhibitor or angiotensin-receptor blocker in a retrospective analysis of patients with heart failure. J Am Heart Assoc 2019; 8: e011089.

64. Anis AH, Sun H, Singh S, Woolcott J, Nosyk B, Brisson M. A cost-utility analysis of losartan versus atenolol in the treatment of hypertension with left ventricular hypertrophy. Pharmacoeconomics 2006; 24: 387-400.

65. Meretoja A, Kaste M, Roine RO. Direct costs of patients with stroke can be continuously monitored on a national level performance, effectiveness, and costs of treatment episodes in stroke (PERFECT Stroke) database in Finland. Stroke 2011; 42: 2007-12.

66. Claesson L, Gosman-Hedstrom G, Johannesson M, Fagerberg B, Blomstrand C. Resource utilization and costs of stroke unit care integrated in a care continuum: a 1-year controlled, prospective, randomized study in elderly patients. The Goteborg 70+ Stroke Study. Stroke 2000; 31: 2569-77.

67. Grieve R, Hutton J, Bhalla A. A comparison of the costs and survival of hospital-admitted stroke patients across Europe. Stroke 2001; 32: 1684-91.

68. Luengo-Fernandez R, Gray AM, Rothwell PM. Population-based study of determinants of initial secondary care costs of acute stroke in the United Kingdom. Stroke 2006; 37: 2579-87.

69. Teng J, Mayo NE, Latimer E. Costs and caregiver consequences of early supported discharge for stroke patients. Stroke 2003; 34: 528-36.

70. The World Bank. Current health expenditure per capita. https://databank.worldbank.org/source/world-development-indicators - Current health expenditure per capita, PPP (current international \$) (access: 1 July 2019). 